\title{
Single-Stage Revision of an Infected Total Hip Replacement Using Antibiotic-Impregnated Bioabsorbable Beads in a Canine Patient
}

\author{
James W. Guthrie ${ }^{1}$ Noel Fitzpatrick ${ }^{1}$ \\ ${ }^{1}$ Department of Orthopaedics and Neurology, Fitzpatrick Referrals, \\ Eashing, United Kingdom of Great Britain and Northern Ireland \\ VCOT Open 2019;2:e5-e12.
}

\author{
Address for correspondence James W. Guthrie, BVM\&S, CertAVP \\ (GSAS), DipECVS, MRCVS, Fitzpatrick Referrals, Halfway Lane, \\ Eashing, Surrey GU7 2QQ, United Kingdom, Ireland \\ (e-mail: jamesg@fitzpatrickreferrals.co.uk).
}

\begin{abstract}
Keywords

- infection

- total hip replacement

- bioabsorbable beads

This report describes a technique and the outcome following surgical revision of a periprosthetic infected total hip replacement (THR) in a single-stage procedure with the use of antibiotic-impregnated bioabsorbable beads. A 6-year-old German Shepherd dog underwent THR 16 months previously, which subsequently became infected. The contaminated femoral and acetabular prostheses were explanted. A femoral window was used to remove the stem and cement. The endosteal surfaces of the femur and acetabulum were reamed to remove periprosthetic tissues. The femoral window was replaced and secured in position with multiple cerclage wires. A 3.5-mm locking plate was applied to the lateral aspect of the femur to prevent fracture of the proximal femoral metaphysis and trochanter. Bioabsorbable beads of calcium sulphate, impregnated with vancomycin and gentamicin, were impacted into the femoral canal followed by an uncemented femoral stem. An uncemented acetabular implant was impacted and additional antibiotic beads placed in the vicinity prior to closure. There has been no evidence of reinfection 5 years postoperatively. Analgesic and antibacterial drugs were not required during this follow-up period; activity is unrestricted with neither lameness nor pain present. Simultaneous revision of both femoral and acetabular periprosthetic infected THR implants was successfully achieved in this patient via a single-stage procedure using uncemented implants and antibiotic-impregnated bioabsorbable beads.
\end{abstract}

\section{Introduction}

Total hip replacement (THR) has become the gold standard surgical procedure to salvage coxofemoral arthrosis in dogs. Periprosthetic infection rates following THR are relatively low, being reported as $1.2,2.2$ and $2.9 \%{ }^{1-3}$ However, when infection does occur it can be catastrophic. Bacterial colonization of the implants results in implant loosening with associated morbidity and reduced function. Treatment of canine patients with septic THR implants usually results in explantation, amputation or euthanasia. ${ }^{2-4}$

To the authors' knowledge, revision of septic THR implants has only been reported in three dogs; one following an experimental study in which only the femoral component was replaced, ${ }^{5}$ another undergoing staged procedures between revision of the acetabular and femoral prostheses ${ }^{6}$ and more recently a report of single-stage revision of all implants using antibiotic-impregnated cement. ${ }^{7}$ In humans, revision of septic THR implants is frequently performed to preserve the prosthetic joint function. This can either be achieved as a staged or single revision procedure. The advantages of a single procedure include reduced patient morbidity, reduced hospitalization, reduced cost and a quicker and improved overall outcome due to fewer surgical procedures and the lack of an interim period. ${ }^{8-11}$ Two-stage received

October 9, 2018

accepted after revision

November 25, 2018
DOI https://doi.org/

10.1055/s-0038-1677523. ISSN 2625-2325. (c) 2019 Georg Thieme Verlag KG
Stuttgart · New York

License terms

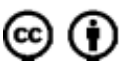


revision involves an initial operation to remove all foreign and infected material, followed by a second procedure to insert new implants. The two-stage approach gained popularity because it was believed to reduce reinfection rates, but recent systematic reviews of the human literature failed to show a difference in the incidence of reinfection when compared with single-stage techniques ( $8.2 \%$ one-stage vs. $7.9 \%$ two-stage) $)^{12,13}$ thus, single-stage revision is now frequently advocated. ${ }^{14}$

A technique to attempt to rid and prevent reoccurrence of infection involves the use of antibiotic-impregnated bioabsorbable materials. ${ }^{15}$ These facilitate the sustained, local release of antimicrobials, which are eventually absorbed, negating the presence of a permanent foreign body. This case report describes the successful revision of all THR implants in a single procedure using the cementless BioMedtrix BFX system (BioMedtrix Inc., Allendale, New Jersey, United States) and antibiotic-impregnated bioabsorbable calcium sulphate beads (Rapidcure, Stimulan: Biocomposites Ltd, Keele, United Kingdom).

\section{Clinical Report}

\section{History}

A 6-year-old, entire male, $29 \mathrm{~kg}$, German Shepherd dog was presented for the assessment of a right pelvic limb lameness and a concurrent discharging sinus tract associated with the right proximal femoral region. The patient had undergone THR on the right side 16 months previously for coxofemoral osteoarthritis secondary to hip dysplasia. Surgery had been routine and achieved with a BioMedtrix Universal Hip implant system (BioMedtrix Inc., Allendale, New Jersey, United States). A size 24 biological ingrowth (BFX; BioMedtrix Inc., Allendale, New Jersey, United States) acetabular component and a cemented (CFX; BioMedtrix Inc., Allendale, New Jersey, United States) size 8 stem component with a $17+3-\mathrm{mm}$ head were implanted. A routine microbiology swab taken at the time of closure was negative for bacterial culture. Routine postoperative clinical assessments at 2, 6 and 12 weeks, along with a radiographical assessment at 6 -week post-surgery were unremarkable, and return to full function was achieved.

Prior to presentation, a discharging sinus had been present for 3 months and treatment with oral amoxicillin-clavulanate (Synulox; Zoetis, London, United Kingdom) and clindamycin (Antirobe; Zoetis, London, United Kingdom) prescribed by the referring veterinarian had failed to resolve the infection. Lameness had been present for 1 week.

\section{Clinical Examination}

A moderate right pelvic limb lameness was appreciable at walk. Significant pain was evident on right coxofemoral joint manipulation and the associated muscles were atrophied. A discharging sinus was located $50 \mathrm{~mm}$ distal to the caudal aspect of the greater trochanter.

\section{Diagnostics}

Radiographs (Elkin Medical Systems Inc., Santa Clara, California, United States) of the pelvis (lateral and ventrodorsal hip extended) and femur (open limb mediolateral and ventrodorsal hip extension with femur parallel to table-top) were obtained under general anaesthesia. Measurements for THR planning were made using digital software templates (Merge Healthcare, eFilm Medical Inc., Milwaukee, Wisconsin, United States). Computed tomography (CT) (Siemens Somatom Emotion; Siemens AG Medical Solutions, Germany) was also performed.

Radiography and CT showed a radiolucent line at the periphery of the cement mantle at the proximal extent of the femur and bone lysis around the proximal third of the cement mantle. Additionally, there was evidence of osseous resorption around the periphery of the acetabular implant (-Fig. 1).

\section{Anaesthesia Protocol}

The patient was premedicated with acepromazine (ACP; Novartis Animal Health UK Ltd., Camberley, United Kingdom) (0.02 $\mathrm{mg} / \mathrm{kg}$ intramuscularly) and methadone (Methadone hydrochloride; Martindale Pharmaceuticals, Romford, United Kingdom) $(0.3 \mathrm{mg} / \mathrm{kg}$ intramuscularly $)$, induced with propofol (Propoflo; Abbott Laboratories Ltd, Maidenhead, United Kingdom) (4mg/kg intravenously), and maintained with isoflurane (Isoflo; Abbott Laboratories Ltd., Maidenhead, United Kingdom) in oxygen. Preoperative analgesia included morphine (Morphine sulfate; Martindale Pharmaceuticals, Romford, United Kingdom) $(0.15 \mathrm{mg} / \mathrm{kg})$ and bupivacaine (Marcain; AstraZeneca, Luton, United Kingdom) $(0.7 \mathrm{mg} / \mathrm{kg})$ into the epidural space. Cefuroxime (Zinacef; GlaxoSmithKline, Middlesex, United Kingdom) (22 mg/kg intravenously) was administered 30 minutes prior to the first incision and every 90 minutes for the duration of the procedure. Methadone (Methadone hydrochloride; Martindale Pharmaceuticals, Romford, United Kingdom) was continued for 12 hours post-surgery and administered every 4 hours.

\section{Surgical Approach}

A craniolateral approach to the right coxofemoral joint ${ }^{16}$ was modified to include dissection down the femoral diaphysis beyond the distal extent of the cement mantle. The tissues comprising the discharging sinus were excised. Total hip replacement was performed using standard BioMedtrix Universal Hip (BioMedtrix Inc., Allendale, New Jersey, United States) BFX components using standard surgical technique according to the manufacturer's recommendations (www. biometrix.com), except for the technical modifications noted below.

\section{Femoral Stem Revision}

Both the femoral and acetabular implants were still tightly adhered, thus requiring significant force to explant them. A sagittal saw was used to create a bevelled edged, rectangular osteotomy along the cranial aspect of the femur to create a window into the medullary canal. ${ }^{17}$ The cement was meticulously excised and the periprosthetic fibrous tissue debrided from the endosteal surface of the femur. The bone window was replaced and seven circumferential cerclage 


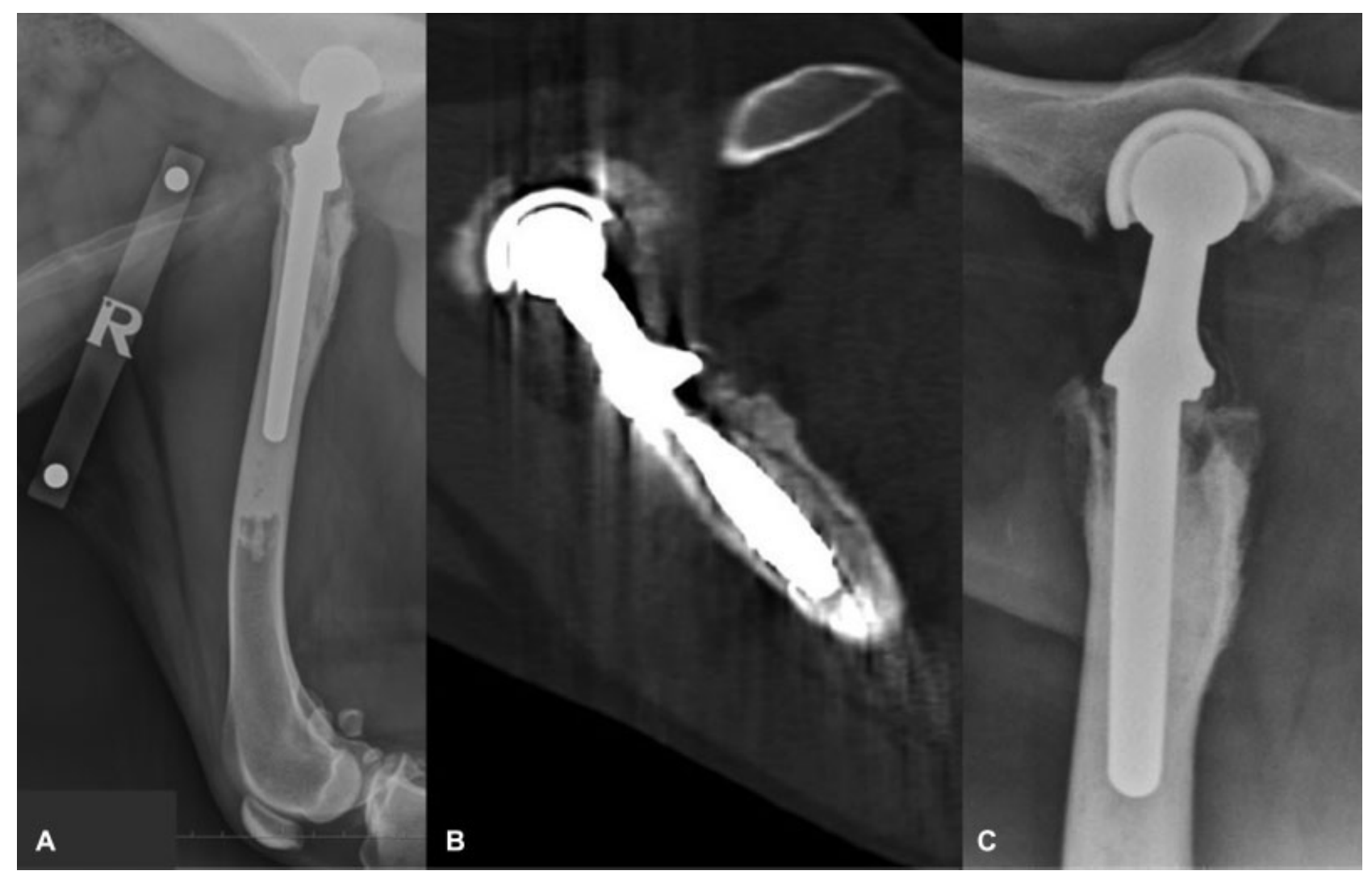

Fig. 1 Preoperative imaging. (A) Mediolateral radiograph of the right femur; (B) Sagittal slice computed tomography through the right proximal femur; (C) Ventrodorsal open limb pelvic radiograph of the right prosthetic coxofemoral joint. Images show bone lysis due to infection at the periphery of the cement mantle at the proximal extent of the femur and immediately distal to the proximal extent of the femoral stem.

wires secured its position. A 3.5-mm SOP plate (String of Pearls; Orthomed, Huddersfield, United Kingdom) was applied to the lateral aspect of the femur to prevent fracture of the proximal femoral metaphysis and greater trochanter. Antibiotic-impregnated bioabsorbable beads of calcium sulphate (Rapidcure, Stimulan; Biocomposites Ltd, Keele, United Kingdom) were formed by mixing $10 \mathrm{cc}$ of the powdered solute with $1000 \mathrm{mg}$ of vancomycin (Vancomycin; Pharmacy, Royal Surrey Hospital, Guildford, Surrey, United Kingdom) powder and $400 \mathrm{mg}(10 \mathrm{~mL})$ solution of gentamicin (Gentamicin; Amdipharm Plc, Basildon, United Kingdom). The substances were mixed for 30 seconds to form a paste, which was spread into the moulds of the bead mat ( - Fig. 2). The beads were allowed to cure for 5 minutes and once solid, compressed into the femoral canal with a broach. A size 10 BFX femoral implant (cobalt chromium stem with a beaded ingrowth surface) was impacted into the femoral canal.

\section{Acetabular Cup Revision}

The original BFX acetabular implant was removed with a combination of a pneumatic spinal burr, an osteotome and an impactor. Care was taken to avoid excessive removal of bone stock and to avoid acetabular bone fracture. Following removal of the original BFX acetabular implant, the underlying acetabular bed was reamed to remove the periprosthetic tissue and a size 26 BFX acetabular implant impacted. All tissues were copiously lavaged with sterile saline throughout the procedure. Further antibiotic beads were positioned around the acetabular cup, proximal femur and femoral neck. Collagen sponges (CollaVET Sponge; Collagen Matrix Inc, Oakland, United States) soaked with $400 \mathrm{mg}$ of gentamicin (Gentamicin; Amdipharm Plc, Basildon, United Kingdom) were placed in the region of the excised sinus tract. Closure was routine and postoperative radiography demonstrated satisfactory implant positioning (- Fig. 3).

\section{Postoperative Care}

A transdermal, sustained-release, fentanyl patch (Duragesic; Janssen-Cilag Ltd., High Wycombe, United Kingdom) $(100 \mu g)$ was applied postoperatively. Oral meloxicam (Metacam; Boehringer Ingelheim, Bracknell, United Kingdom) $(0.1 \mathrm{mg} /$ $\mathrm{kg}$ ) was administered once daily for 4 weeks. A pure growth of a Staphylococcus species with no antibiotic resistance was obtained following prolonged culture of the infected implants. Oral clindamycin (Synulox: Zoetis, London, United Kingdom) (11 mg/kg) was continued twice daily for 3 weeks postoperatively. Exercise was restricted to controlled lead walks of increasing duration for the first 12 weeks. Postoperative physiotherapy was commenced after 2 weeks and hydrotherapy initiated after 6 weeks.

\section{Outcome}

Lameness was mild after 6 weeks and resolved by 12 weeks. Re-examination at 6,12 and 48 months revealed no lameness and the joint was non-painful in all phases of motion. There was no evidence of implant loosening at either time point on 


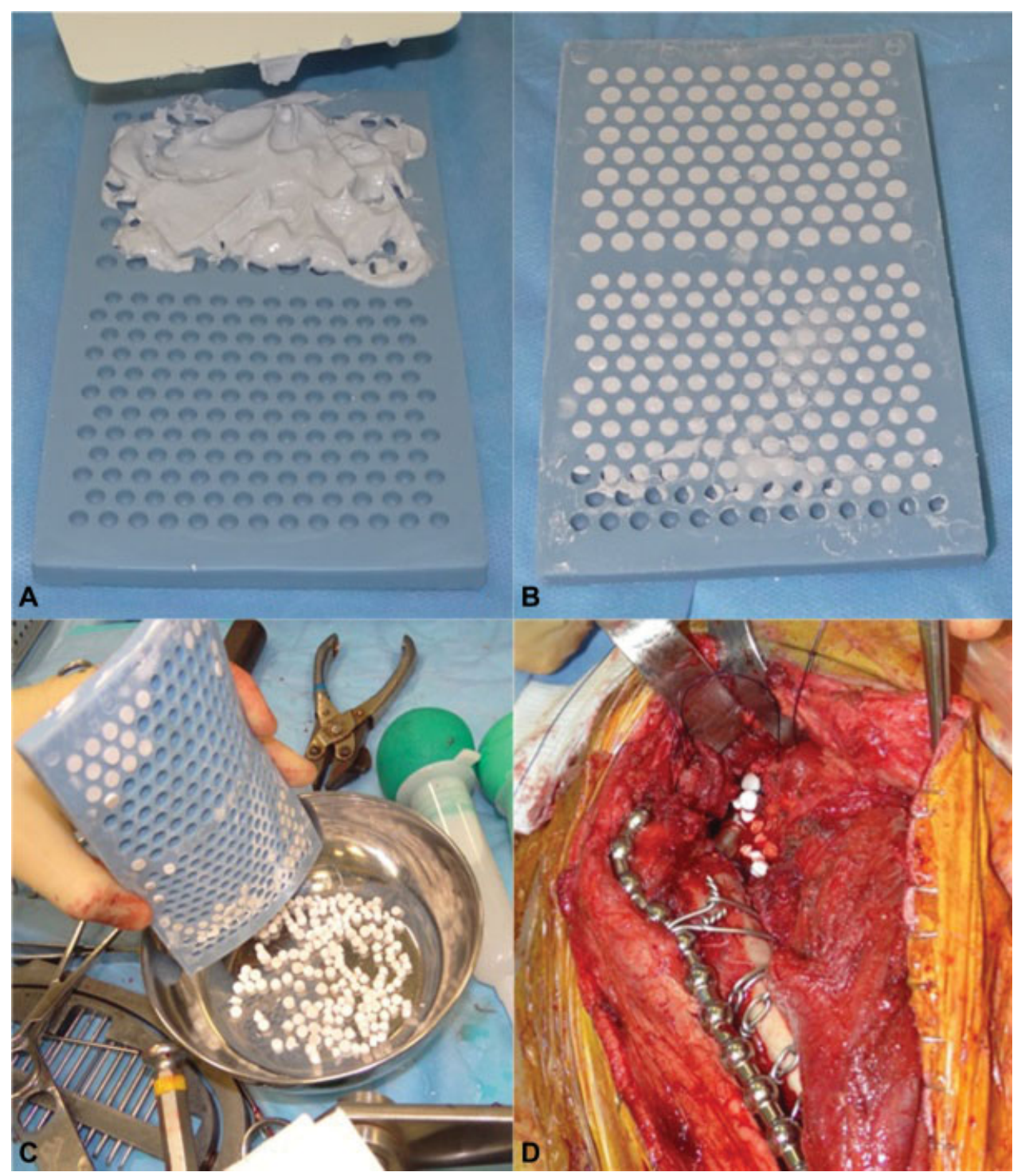

Fig. 2 Preparation and application of antibiotic-impregnated bioabsorbable beads. (A) Antibiotic solution mixed with bead solute to form paste; (B) Paste applied to mould and allowed to cure; (C) Cured beads removed from mould; (D) Placement of bioabsorbable beads around the prosthetic femoral neck.

physical or radiographical examination (-Fig. 4). The femoral stem subsided $7.5 \mathrm{~mm}$ prior to radiography 6 weeks post-surgery ( - Fig. 4); no further subsidence occurred after this time. Radiography demonstrated complete absorption of the antibiotic-impregnated beads. At 12 and 48 months of post-surgery, gait analysis was performed by measuring ground reaction forces of each pelvic limb at walk using a force plate and the peak vertical forces were calculated (BioWare 5.1.1; Kistler Instruments Ltd., Hook, United Kingdom). Peak vertical forces were normalized to body mass. The force plate data demonstrated a symmetry index of 2.5 (12 months) and 3.1 (48 months) which is considered normal. ${ }^{18} \mathrm{~A}$ telephone interview with the owner 60 months following revision surgery revealed the patient was still free of lameness. Analgesia and antibiotic medications were not required and activity has been unrestricted. There have been no complications or indications of recurrent sepsis for 5 years since the revision surgery.

\section{Discussion}

Total hip replacement periprosthetic infection and subsequent implant loosening are infrequently reported in the veterinary literature, occurring in $1.2,2.2$ and $2.9 \%$ of 256,97 and 221 procedures respectively. ${ }^{1-3}$ Infection can become established from intraoperative contamination, local extension of wound infection or haematogenous spread. ${ }^{19}$ In our case, infection was diagnosed based on radiographical and CT findings of periprosthetic lucency and periostitis, as well as the presence of a discharging sinus tracking from the location of the affected coxofemoral joint found on physical examination. An attempt to obtain an antimicrobial culture preoperatively was not made; in the human literature regarding $\mathrm{THR}^{20}$ it has been documented that infections contain a variety of colony variants and conventional culture techniques frequently do not detect the specific causative organism. ${ }^{21}$ Subsequently, bacterial growth from this case 


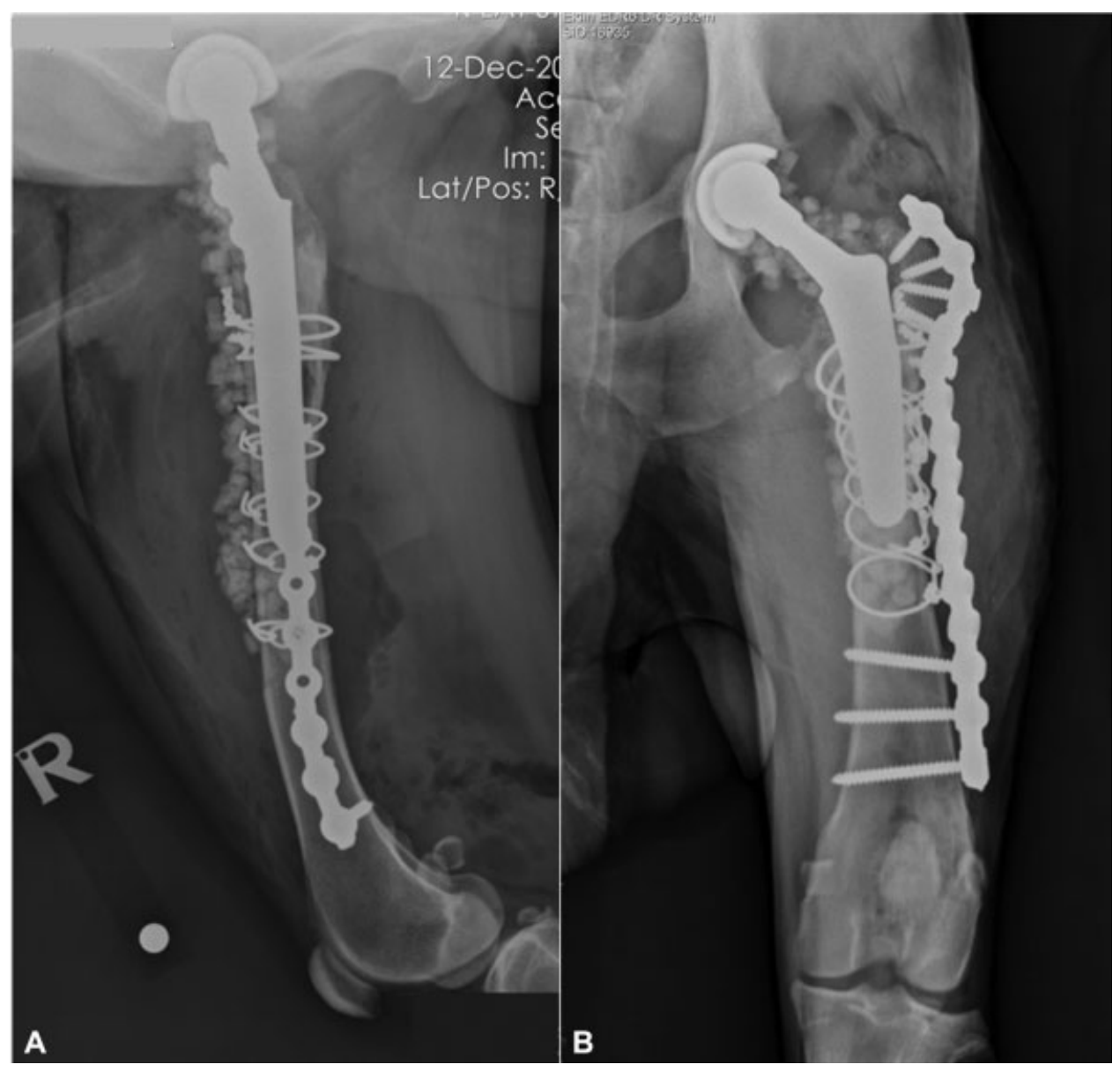

Fig. 3 Immediate postoperative imaging. (A) Mediolateral radiograph of the right femur; (B) Craniocaudal radiograph of the right femur. The antibiotic-impregnated bioabsorbable beads can be seen surrounding the femur and acetabulum.

was only possible following prolonged culture from one of three samples acquired intraoperatively. Should culture fail, clinicians could use polymerase chain reaction to identify bacterial material. ${ }^{22}$ The use of an antimicrobial such as vancomycin without a positive culture and sensitivity is controversial and the authors recommend readers consider their antimicrobial choices responsibly. Our choice of antibiotic medications was based on four key factors:

1. we selected synergistic antimicrobials, ${ }^{23}$

2. antimicrobials that were frequently used in the management of human periprosthetic infections at the time (2012) our patient was treated, ${ }^{24}$

3. antimicrobials that have had documented use by the calcium sulphate bead manufacturer, ${ }^{25}$

4. and based on microbiology data from surgical site infections at our practice. ${ }^{26}$

During the original THR procedure for the dog in this report, an intraoperative swab was taken for microbiology, which failed to culture any organisms. No septic source for the periprosthetic infection 13 months later was observed, suggestive of haematogenous bacterial spread or the presence of residual bacteria from the original procedure. The bacterial flora most commonly cultured from explanted septic THR implants in dogs are Staphylococcus species. ${ }^{1}$
This is consistent with the infectious agent present in our patient. The laboratory was unable to identify the exact Staphylococcus species as at the time they were unable to provide more advanced biochemical tests, such as DNase testing, for coagulase-negative Staphylococcus species.

We used cementless implants because both uncemented femoral ${ }^{27}$ and uncemented acetabular ${ }^{28}$ implants are associated with improved outcomes for revision of septic human THR. Additionally, polymethyl methacrylate (PMMA) bone cement is an ideal substrate for bacterial attachment and replication of sessile bacterial phenotypes. ${ }^{29}$ To reduce the risk of colonization of PMMA cement, the cement can be loaded with antibiotic medications. One case of single-stage revision of a canine THR using vancomycin and micro-silver antimicrobial powder impregnated cement has recently been reported. ${ }^{7}$

To reduce the risk of re-infection, we implanted antibioticimpregnated bioabsorbable materials. These allow local release of antibiotic medications and are gradually absorbed by the body so that no permanent foreign tissue remains. High concentrations of local antibiotic medications can be provided, which can increase the penetration of biofilm and necrotic tissue as well as increasing the efficacy if their activity is concentration dependent. ${ }^{30}$ Although local concentrations are high, serum concentrations are frequently 


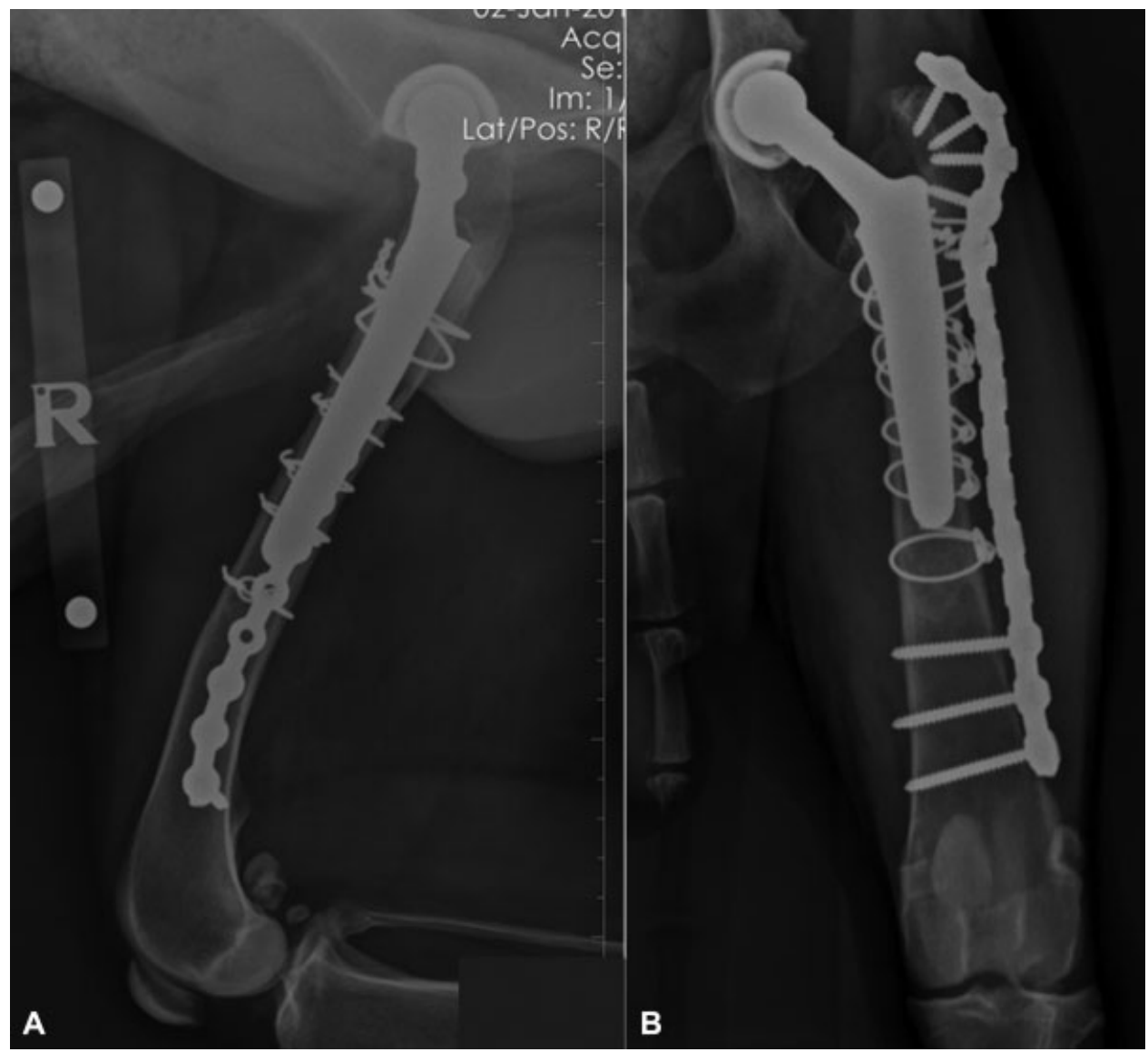

Fig. 4 Forty-eight months postoperative imaging. (A) Mediolateral radiograph of the right femur; (B) Craniocaudal radiograph of the right femur. The antibiotic-impregnated bioabsorbable beads have been absorbed and no indication of sepsis is present.

low or undetectable, decreasing the risk of drug reactions and systemic toxicosis. Implantation of antibiotic medications during surgery also negates the risk for non-compliance of medication administration postoperatively, which can be a problem in some veterinary patients. ${ }^{31}$

The antibiotic-impregnated bioabsorbable calcium sulphate beads are completely absorbed within 4 weeks, ${ }^{32}$ so a second surgery for removal is not necessary. These beads are osteoconductive and provide a scaffold for new bone ${ }^{33}$; therefore, using them within the femoral medullary canal would prevent dead space formation while they were absorbed. The beads we used are a synthetic hemihydrate form of calcium sulphate and are processed to produce a product free from potential toxic impurities. ${ }^{25}$ During in vitro testing, elution of antibiotic medications from these beads has been shown to be predictable and last at least 28 days, ${ }^{34}$ but reported clinical experience has revealed a longer time frame if the beads are not completely covered by soft tissue. $^{35}$ The beads cure at a low temperature, thus allowing heat-sensitive antibiotic medications to be mixed. The cured beads are soft after hydration and are less likely to scratch implants or the joint surface. ${ }^{25}$ These calcium sulphate beads have been used in human patients for revision surgery of both total hip and total knee replacements with a reported re-infection rate of $2.4 \% .^{33}$ The use of these calcium sulphate beads has not previously been reported in veterinary patients to deliver local antibiotic medications.
Collagen sponges are another absorbable material, which can be used to provide local delivery of antibiotic medications. ${ }^{31}$ These have been used to treat a range of canine orthopaedic-related infections. ${ }^{36,37}$ These are absorbed more quickly than the calcium sulphate beads, so were utilized within the draining tract to mirror the faster healing and tissue ingrowth time of soft tissues. In humans, discharging sinuses have been shown not to be a contraindication to single-stage revision of THR. ${ }^{8}$ The antibiotic medications we used perioperatively were broad spectrum and known to have activity against the most commonly cultured bacterial species from infected canine orthopaedic implants; postoperative antibiotic medication was based on microbiology culture and sensitivity results. We administered postoperative oral antibiotic medications for 3 weeks; this is shorter than protocols frequently employed by human surgeons, but there is currently debate within the human literature regarding the ideal protocol. ${ }^{24}$ Consequently, longer periods of antibiotic therapy may be considered.

As the femoral stem subsided $7.5 \mathrm{~mm}$, there may have been the possibility to up-size the femoral implant to a size $11 \mathrm{BFX}$. However, due to the lysis of the proximal femur, and the creation of the 'coffin-lid' window, there was great concern that broaching and impacting a larger stem could result in a fracture to the femur. Although the stem subsided within the first 6 weeks following surgery, no clinical implications of this were noted. 
Although this is a report of only one case, it highlights that infected THR implants in dogs can successfully be replaced in a single procedure. Development of optimal exchange protocols as well as predictive factors to obtain successful outcomes will only become evident following larger case series and are therefore indicated. Further randomized and prospective clinical trials are warranted to fully assess their effect and merit, but our report demonstrates that antibioticimpregnated bioabsorbable calcium sulphate beads can be useful to improve management of implant infections in veterinary orthopaedics.

\section{Author Contribution}

Both authors contributed to conception of study, study design, acquisition of data and data analysis and interpretation. Both of them also drafted, revised and approved the submitted manuscript.

\section{Conflict of Interest}

None declared.

\section{References}

1 Dyce J, Wisner ER, Wang Q, Olmstead ML. Evaluation of risk factors for luxation after total hip replacement in dogs. Vet Surg 2000;29 (06):524-532

2 Bergh MS, Gilley RS, Shofer FS, Kapatkin AS. Complications and radiographic findings following cemented total hip replacement: a retrospective evaluation of 97 dogs. Vet Comp Orthop Traumatol 2006;19(03):172-179

3 Olmstead ML, Hohn RB, Turner TM. A five-year study of 221 total hip replacements in the dog. J Am Vet Med Assoc 1983;183(02): 191-194

4 Girling SL, Innes JF. Infection of a total hip prosthesis in a dog caused by Achromobacter(Alcaligenes) xylosoxidans. J Small Anim Pract 2006;47(12):747-750

5 Massat BJ, Miller RT, DeYoung BA, Schiller RA, Aberman HM, DeYoung DJ. Single-stage revision using an uncemented, porouscoated, anatomic endoprosthesis in two dogs: case report. Vet Surg 1998;27(03):268-277

6 Torres BT, Budsberg SC. Revision of cemented total hip arthroplasty with cementless components in three dogs. Vet Surg 2009; 38(01):81-86

7 Ficklin MG, Kowaleski MP, Kunkel KA, Suber JT. One-stage revision of an infected cementless total hip replacement. Vet Comp Orthop Traumatol 2016;29(06):541-546

8 Raut VV, Siney PD, Wroblewski BM. One-stage revision of infected total hip replacements with discharging sinuses. J Bone Joint Surg Br 1994;76(05):721-724

9 Ure KJ, Amstutz HC, Nasser S, Schmalzried TP. Direct-exchange arthroplasty for the treatment of infection after total hip replacement. An average ten-year follow-up. J Bone Joint Surg Am 1998; 80(07):961-968

10 Klouche S, Sariali E, Mamoudy P. Total hip arthroplasty revision due to infection: a cost analysis approach. Orthop Traumatol Surg Res 2010;96(02):124-132

11 Lange J, Troelsen A, Thomsen RW, Søballe K. Chronic infections in hip arthroplasties: comparing risk of reinfection following onestage and two-stage revision: a systematic review and metaanalysis. Clin Epidemiol 2012;4:57-73

12 Leonard HA, Liddle AD, Burke O, Murray DW, Pandit H. Single- or two-stage revision for infected total hip arthroplasty? A systema- tic review of the literature. Clin Orthop Relat Res 2014;472(03): 1036-1042

13 Kunutsor SK, Whitehouse MR, Blom AW, Beswick AD; INFORM Team. Re-infection outcomes following one- and two-stage surgical revision of infected hip prosthesis: a systematic review and meta-analysis. PLoS One 2015;10(09):e0139166

14 Zeller V, Lhotellier L, Marmor S, et al. One-stage exchange arthroplasty for chronic periprosthetic hip infection: results of a large prospective cohort study. J Bone Joint Surg Am 2014;96(01):e1

15 Ham K, Griffon D, Seddighi M, Johnson AL. Clinical application of tobramycin-impregnated calcium sulfate beads in six dogs (20022004). J Am Anim Hosp Assoc 2008;44(06):320-326

16 Piermattei DL, Johnson KA. An Atlas of Surgical Approaches to Bones and Joints of the Dog and Cat. St. Louis: Elsevier Saunders; 2004

17 Dyce J, Olmstead ML. Removal of infected canine cemented total hip prostheses using a femoral window technique. Vet Surg 2002; 31(06):552-560

18 Voss K, Imhof J, Kaestner S, Montavon PM. Force plate gait analysis at the walk and trot in dogs with low-grade hindlimb lameness. Vet Comp Orthop Traumatol 2007;20(04):299-304

19 Spangehl MJ, Younger AS, Masri BA, et al. Diagnosis of infection following total hip arthroplasty. J Bone Joint Surg Am 1997; 79:1578-1588

20 Winkler $\mathrm{H}$. Rationale for one stage exchange of infected hip replacement using uncemented implants and antibiotic impregnated bone graft. Int J Med Sci 2009;6(05):247-252

21 Fux CA, Stoodley P, Hall-Stoodley L, Costerton JW. Bacterial biofilms: a diagnostic and therapeutic challenge. Expert Rev Anti Infect Ther 2003;1(04):667-683

22 Mariaux S, Tafin UF, Borens O. Diagnosis of persistent infection in prosthetic two-stage exchange: PCR analysis of sonication fluid from bone cement spacers. J Bone Jt Infect 2017;2(04):218-223

23 Bertazzoni Minelli E, Della Bora T, Benini A. Different microbial biofilm formation on polymethylmethacrylate (PMMA) bone cement loaded with gentamicin and vancomycin. Anaerobe 2011;17(06):380-383

24 Senthi S, Munro JT, Pitto RP. Infection in total hip replacement: meta-analysis. Int Orthop 2011;35(02):253-260

25 Stimulan Rapid Cure. Available at: http://www.biocomposites. com/our-products/stimulan/. Accessed March 2, 2018

26 Diribe O, Thomas S, AbuOun M, Fitzpatrick N, La Ragione R. Genotypic relatedness and characterization of Staphylococcus pseudintermedius associated with post-operative surgical infections in dogs. J Med Microbiol 2015;64:1074-1081

27 Rothman RH, Cohn JC. Cemented versus cementless total hip arthroplasty. A critical review. Clin Orthop Relat Res 1990;(254): 153-169

28 Park DK, Della Valle CJ, Quigley L, Moric M, Rosenberg AG, Galante JO. Revision of the acetabular component without cement. A concise follow-up, at twenty to twenty-four years, of a previous report. J Bone Joint Surg Am 2009;91(02):350-355

29 Gristina AG, Naylor P, Myrvik Q. Infections from biomaterials and implants: a race for the surface. Med Prog Technol 1988-1989:14 (3-4):205-224

30 Diefenbeck M, Mückley T, Hofmann GO. Prophylaxis and treatment of implant-related infections by local application of antibiotics. Injury 2006;37(Suppl 2):S95-S104

31 Hayes G, Moens N, Gibson T. A review of local antibiotic implants and applications to veterinary orthopaedic surgery. Vet Comp Orthop Traumatol 2013;26(04):251-259

32 Agarwal S, Healey B. The use of antibiotic impregnated absorbable calcium sulphate beads in management of infected joint replacement prostheses. J Arthrosc Jt Surg 2014;2:72-75

33 McPherson EJ, Dipane MV, Sherif SM. Dissolvable antibiotic beads in treatment of periprosthetic joint infection and revision arthroplasty. The use of synthetic pure calcium sulfate (Stimulan) 
e12 Antibiotic-Impregnated Bioabsorbable Beads for Revision of Infected THR Guthrie, Fitzpatrick

impregnated with vancomycin \& tobramycin. Reconstr Rev 2013; 3:32-43

34 Kanellakopoulou K, Panagopoulos P, Giannitsioti E, et al. In vitro elution of daptomycin by a synthetic crystallic semihydrate form of calcium sulfate, Stimulan. Antimicrob Agents Chemother 2009; 53(07):3106-3107

35 Gauland C. Managing lower-extremity osteomyelitis locally with surgical debridement and synthetic calcium sulfate antibiotic tablets. Adv Skin Wound Care 2011;24(11):515-523
36 Owen MR, Moores AP, Coe RJ. Management of MRSA septic arthritis in a dog using a gentamicin-impregnated collagen sponge. J Small Anim Pract 2004;45(12):609 612

37 Renwick AI, Dennis R, Gemmill TJ. Treatment of lumbosacral discospondylitis by surgical stabilisation and application of a gentamicin-impregnated collagen sponge. Vet Comp Orthop Traumatol 2010;23(04):266-272 